Delft University of Technology

\title{
Silicon Nitride MOMS Oscillator for Room Temperature Quantum Optomechanics
}

Serra, Enrico; Morana, Bruno; Borrielli, Antonio; Marin, Francesco; Pandraud, Gregory; Pontin, Antonio; Prodi, Giovanni Andrea; Sarro, Pasqualina M.; Bonaldi, Michele

DOI

10.1109/JMEMS.2018.2876593

Publication date

2018

Document Version

Final published version

Published in

Journal of Microelectromechanical Systems

\section{Citation (APA)}

Serra, E., Morana, B., Borrielli, A., Marin, F., Pandraud, G., Pontin, A., Prodi, G. A., Sarro, P. M., \& Bonaldi, M. (2018). Silicon Nitride MOMS Oscillator for Room Temperature Quantum Optomechanics. Journal of Microelectromechanical Systems, 27(6), 1193-1203. [8520914]. https://doi.org/10.1109/JMEMS.2018.2876593

Important note

To cite this publication, please use the final published version (if applicable).

Please check the document version above. 
Green Open Access added to TU Delft Institutional Repository

'You share, we take care!' - Taverne project

https://www.openaccess.nl/en/you-share-we-take-care

Otherwise as indicated in the copyright section: the publisher is the copyright holder of this work and the author uses the Dutch legislation to make this work public. 


\title{
Silicon Nitride MOMS Oscillator for Room Temperature Quantum Optomechanics
}

\author{
Enrico Serra ${ }^{\circledR}$, Bruno Morana, Antonio Borrielli, Francesco Marin ${ }^{\circledR}$, Gregory Pandraud, Antonio Pontin, \\ Giovanni Andrea Prodi, Pasqualina M. Sarro, Fellow, IEEE, and Michele Bonaldi ${ }^{\circledR}$
}

\begin{abstract}
Optomechanical SiN nano-oscillators in high-finesse Fabry-Perot cavities can be used to investigate the interaction between mechanical and optical degree of freedom for ultra-sensitive metrology and fundamental quantum mechanical studies. In this paper, we present a nano-oscillator made of a high-stress round-shaped $\mathrm{SiN}$ membrane with an integrated on-chip 3-D acoustic shield properly designed to reduce mechanical losses. This oscillator works in the range of $200 \mathrm{kHz}$ to $5 \mathrm{MHz}$ and features a mechanical quality factor of $Q \simeq 10^{7}$ and a Q-frequency product in excess of $6.2 \times 10^{12} \mathrm{~Hz}$ at room temperature, fulfilling the minimum requirement for quantum ground-state cooling of the oscillator in an optomechanical cavity. The device is obtained by MEMS deep reactive-ion etching (DRIE) bulk micromachining with a two-side silicon processing on a silicon-on-insulator wafer. The microfabrication process is quite flexible such that additional layers could be deposited over the SiN membrane before the DRIE steps, if required for a sensing application. Therefore, such oscillator is a promising candidate for quantum sensing applications in the context of the emerging field of quantum technologies.

[2018-0186]
\end{abstract}

Index Terms-MOMS oscillator, quantum optomechanics, $\mathrm{SiN}$ thin membrane, reactive ion etching.

\section{INTRODUCTION}

$\mathbf{T}$ HE study of quantum phenomena is of outmost importance for the understanding and assessment of modern physical theories and for the development of radical new technologies. Light is typically at the basis of many of the experiments aiming to these studies. For instance, in cavity

Manuscript received August 9, 2018; revised October 10, 2018; accepted October 13, 2018. Date of publication November 2, 2018; date of current version November 29, 2018. This work was supported in part by the QuaSeRT Project under Grant QuantERA ERA-NET, in part by the Quantum Technologies through the European Union's Horizon 2020 Programme, and in part by the Istituto Nazionale di Fisica Nucleare under the HUMOR Project. Subject Editor S. Merlo. (Corresponding author: Enrico Serra).

E. Serra is with the Istituto Nazionale di Fisica Nucleare, TIFPA, 38123 Trento, Italy (e-mail: enrico.serra@tifpa.infn.it).

B. Morana, G. Pandraud, and P. M. Sarro are with ECTM-EKL, Delft University of Technology, 2628 Delft, The Netherlands (e-mail: b.morana@tudelft.nl; g.pandraud@tudelft.nl; p.m.sarro@tudelft.nl).

A. Borrielli and M. Bonaldi are with the Nanoscience-Trento-FBK Division, Institute of Materials for Electronics and Magnetism, 38123 Trento, Italy (e-mail: borrielli@fbk.eu; mbonaldi@fbk.eu).

F. Marin and A. Pontin are with the Dipartimento di Fisica e Astronomia and LENS, Università di Firenze, 50121 Florence, Italy, also with INFN Sezione di Firenze, 50019 Sesto Fiorentino (FI), Italy, and also with CNR-INO, 50125 Florence, Italy (e-mail: marin@fi.infn.it; antonio.pontin@gmail.com).

G. A. Prodi is with the Dipartimento di Fisica, Università di Trento, 38123 Trento, Italy (e-mail: giovanniandrea.prodi@unitn.it).

Color versions of one or more of the figures in this paper are available online at http://ieeexplore.ieee.org.

Digital Object Identifier 10.1109/JMEMS.2018.2876593 optomechanics [1], [2], light is coupled to resonant mechanical systems inserted in a optical cavity to enable studies on quantum theory and quantum gravity. Among these we mention the sensing of forces or fields at the ultimate limits imposed by quantum mechanics [3], [4], and experiments testing the foundation of physics, i.e., fixing significant constraints for the development of quantum gravity theories [5], [6].

Effects related to the quantum fluctuations of the radiation pressure are not easily detected because of classical noise sources, like the Brownian thermal noise and spurious coupling of the optomechanical system to the environment. As the number of quantum-coherent oscillations in the presence of thermal decoherence scales with the $\mathrm{Q} \times \mathrm{f}$ product (mechanical quality factor $\times$ oscillator frequency), this parameter is commonly used as a figure of merit for evaluating the oscillator's performance in optomechanics [2]. For this reason Silicon nitride ( $\mathrm{SiN})$ micro- and nano-mechanical membrane resonators have attracted a lot of attention due to their exceptionally high-Q factors [7], [8]. Systems based on a membrane oscillator have shown for the first time the mechanical effect of the quantum noise in the light [9] and one of the first observations of pondero-motive light squeezing [10]. Moreover, high-Q SiN membranes are interesting because they can be used in collective optotomechanics, for the realization of entangled states between mechanical modes [11], or in systems working in the anti-squashing regime of positive feedback for enhanced sideband cooling [12].

In many cases the oscillators consist of a commercial free-standing high-stress silicon nitride ( $\mathrm{SiN}$ ) membrane supported by a silicon frame, where mechanical quality factors up to many millions can be in principle obtained [13] thanks to the large tensile stress (close to $1 \mathrm{GPa}$ ). However, in these systems the mechanical loss is strongly dependent on the mounting, especially for the low frequency modes. This causes low Q-factors with values scattered between $10^{4}$ and $10^{6}$ [14]. Different approaches have been used to isolate the $\mathrm{SiN}$ membrane from its support. For instance in [11] a photonic bandgaps allowed the observation of ponderomotive squeezing at moderate cryogenic temperatures, while in [15]-[17] the resonating part is decoupled from its substrate by highaspect-ratio SiN trampolines. These achievements motivate the research on customized SiN resonators for optomechanics, using MEMS wafer-scale approaches and with a precise control of the overall dissipation mechanisms [18]. In fact, the mechanical dissipation in an oscillator determines the thermal fluctuation noise, and quantum behavior can emerge 


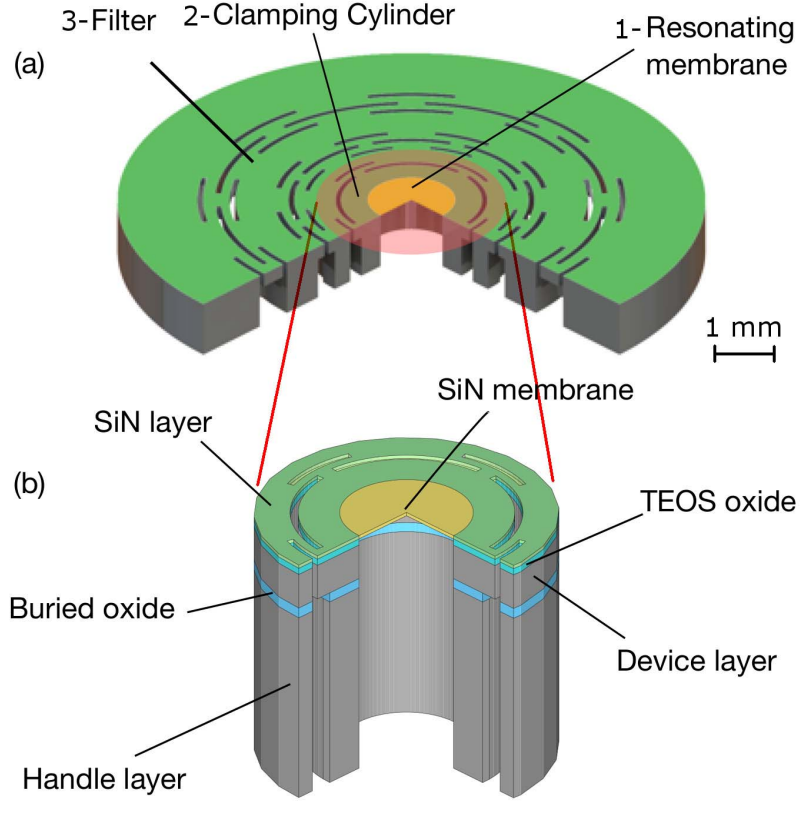

Fig. 1. (a) View of the SiN circular membrane oscillator. The main oscillator 1 ( $\mathrm{SiN}$ membrane) is in the center and surrounded by the cylinder 2 and loss-shield structure (filter) 3. (b) Detailed view of the central part of the oscillator showing the SOI wafer and thin film layers (not to scale).

only if the thermal noise force is lower than noise of quantum origin, such as radiation pressure shot-noise.

We have recently proposed a novel coupled oscillators model for the mechanical losses in a membrane oscillator [19]. This takes into account the mutual interaction between the membrane and the frame in a recoil losses analysis. We were able to design an effective shield for the losses for all mechanical modes also in the low frequency range. In this work, we present the design and microfabrication process of a MOMS oscillator built following these design rules, where a circular SiN membrane [20] is integrated on-chip with a silicon loss-shield. The shield works as isolation stage and protects the oscillator from the mechanical decoherence induced by the thermal bath. The device works from room to cryogenic temperatures, and reaches high $\mathrm{f} \times \mathrm{Q}$ values for all resonant modes in the measured bandwidth [0.2-5] MHz. Silicon double-side deep-RIE etching on a Silicon-On-Insulator (SOI) wafer were done with wet-etch HF steps for removing sacrificial oxide and $\mathrm{SiN}$ membrane release.

\section{RESONATOR DESIGN}

The proposed device consist of round-shaped high-stress non-stoichiometric SiN membrane (with tensile intrinsic stress of $0.830 \mathrm{GPa}$ ), deposited by Low-Pressure Chemical Vapor Deposition (LPCVD), of thickness around $100 \mathrm{~nm}$. This membrane interacts by the action of the radiation pressure, with a $1064 \mathrm{~nm}$ laser beam. To improve the quality factor and to reject vibrational noise from the environment, a mechanical filtering stage is integrated on-chip by exploiting double side Deep Reactive-Ion Etching (DRIE). A view of the device is shown in Fig. 1. The design of the SiN MOMS oscillator comprises of three main parts: (1) the resonating thin silicon nitride ( $\mathrm{SiN}$ ) nanomembrane, (2) the loss-shield working as intermediate filter (made of a clamping hollow cylinder and flexural/torsional springs), (3) the outer silicon frame that connects the oscillator to the sample holder. Fig. 1b shows a detailed view of the cross section of the central part of the system, highlighting the thin-film layers and the SOI wafer structure. In the subsections II-A, II-B, II-C we focus on how to minimize the thermal noise effects by reducing the mechanical dissipations of the substrate with a loss shield. In subsection II-D we discuss how to limit the detrimental effects on Q-factor due to the oxide layer even at liquid-He temperatures.

The Power Spectral Density (PSD) of the thermal noise force is $S_{T h}=4 k_{B} T \frac{m \omega}{Q}$, where $m$ is the mass of the oscillator, $\omega$ its angular frequency, and $T$ the temperature. The quality factor $Q$ is simply related to the fraction of mechanical energy loss per oscillation cycle, called $\phi$, as: $Q^{-1}=\phi \equiv \frac{\Delta W_{t}}{2 \pi W_{t}}$, with $W_{t}$ the energy stored in the resonant mode and $\Delta W_{t}$ the energy loss per cycle. As said above, the figure of merit which is the product of mechanical quality factor $Q$ and frequency $f$ is used as object function in the device optimization process. Indeed, this figure of merit determines the number of coherent oscillations in the presence of thermal fluctuations, and the minimum requirement for room-temperature quantum optomechanics is $Q \times f>6.2 \mathrm{THz}$ [2].

\section{A. Total Losses}

The energy loss in a $\mathrm{SiN}$ membrane is limited by intrinsic loss, that is the imaginary component in the stress-strain relation. This loss sets the ultimate limit $Q_{i n t}$ for the mode's $Q$-factors, but when the membrane is part of a complex device, different loss channels add up according to this simple relation:

$$
Q_{T O T}^{-1}=\phi_{T O T}=\phi_{\text {int }}+\phi_{\text {clam }}+\phi_{\text {gas }}+\phi_{T E D}
$$

Here $\phi_{\text {int }}$ is the internal damping of silicon nitride, $\phi_{\text {clam }}$ accounts for the clamping loss, $\phi_{\text {gas }}$ describes viscous damping arising from gas surrounding the membrane, and $\phi_{T E D}$ is the thermoelastic damping. We point out that, in a highly stressed membrane oscillator, the total $Q$-factor is also proportional to the internal stress of the SiN membrane, as calculated in detail in [13], [21]. In an approximate view, the effect of internal stress can be understood by noting that the shape of the normal mode is not affected by the stress (especially in the case of low order modes), so that the energy lost per cycle due to internal damping remains unchanged. On the other hand the energy stored in the mode increases for the tension applied to the membrane, reducing the fraction of mechanical energy lost per oscillation cycle $\frac{\Delta W_{t}}{2 \pi W_{t}}$. Currently, given that the internal stress cannot be much larger than $1 \mathrm{GPa}$, the technological efforts are directed to reduce contributions from each of the loss channels.

In our MOMS device we can neglect the gas damping $\phi_{\text {gas }}$ because the oscillator is employed in a High Vacuum setup, and the thermoelastic damping of the $\mathrm{SiN}$ layer is orders of magnitude lower than the other loss mechanisms. Therefore the most relevant contribution is the clamping 
loss $\phi_{\text {clam }}$, that depends on the mechanical impedance seen at the support. In fact, as observed for instance by Morse [22] for the case of a string supported by a sounding board, the damping depends on the mechanical impedance of the support. Instead, in the special case of infinitely rigid and massive support, the damping of the oscillating part is determined only by its intrinsic dissipation. In case of highly stressed membranes, the typical resonant frequency is around $1 \mathrm{MHz}$. In this frequency range, the silicon die cannot be considered as a rigid body but its full modal response must be considered in evaluating its admittance. This because a support resonating at the same frequency of the membrane can be very effective in absorbing the mechanical energy. For this reason we have developed a theoretical model [19] where we evaluate the loss when the membrane and support are fullycoupled, i.e. allowing for the transfer of energy in both ways. In the next section we evaluate the clamping loss when the membrane and the silicon frame are coupled structures, and we show how the loss can be reduced by using mechanical insulation stages i.e. a loss-shield structure.

\section{B. Coupled System Losses}

For a precise evaluation of total loss, we consider two-way energy transfer between membrane and its support. The coupling is originated from the recoil forces or torques of the structure surrounding the vibrating SiN membrane. The damping originating from recoil forces has been first evaluated by Saulson [23] in the analysis of two cascaded pendula used as mechanical filters in Gravitational Wave detectors. If we model the membrane of mass $M_{m}$ and the wafer of mass $M_{w}$ as two coupled harmonic oscillator, with $\omega_{m}$ and $\omega_{w}$ the resonant frequencies of the uncoupled parts, the effective loss angle $\bar{\phi}_{m}$ of the membrane in the coupled system is:

$$
\bar{\phi}_{m}\left(\omega_{m}\right)=\phi_{m}+\phi_{w} \frac{M_{m}}{M_{w}} \frac{\omega_{w}^{2} \omega_{m}^{2}}{\left(\omega_{w}^{2}-\omega_{m}^{2}\right)^{2}}, \quad \omega_{w} \neq \omega_{m}
$$

where $\phi_{m}, \phi_{w}$ are the intrinsic loss factor of the membrane and the wafer, respectively. This equation shows that in the case of a massive, rigid support with a small intrinsic dissipation $\left(\phi_{w} \simeq 0\right)$, the dissipation of the coupled system is essentially determined by the intrinsic loss in the membrane. But if the two parts are at resonance, i.e. $\omega_{w} \simeq \omega_{m}$, an approximated solution, valid for the case $\phi_{m} \ll 1,10^{2}<\phi_{w}^{-1}<10^{5}$ and $M_{w} / M_{m} \gg \frac{1}{\phi_{w}^{2}}$, gives:

$$
\bar{\phi}_{m} \simeq \phi_{m}+\frac{1}{\phi_{w}^{2}} \frac{M_{m}^{2}}{M_{w}^{2}}, \quad \omega_{w} \simeq \omega_{m}
$$

and we see that the loss of the coupled membrane will grow as some mechanical energy leaks toward the supporting wafer.

\section{Shielding Recoil Losses}

To overcome the loss due to the coupling between the wafer and the resonating membrane, we introduce a third body with a known dynamical impedance (loss-shield stage) that can effectively decouple the membrane from the support. This intermediate stage is also effective in filtering-out
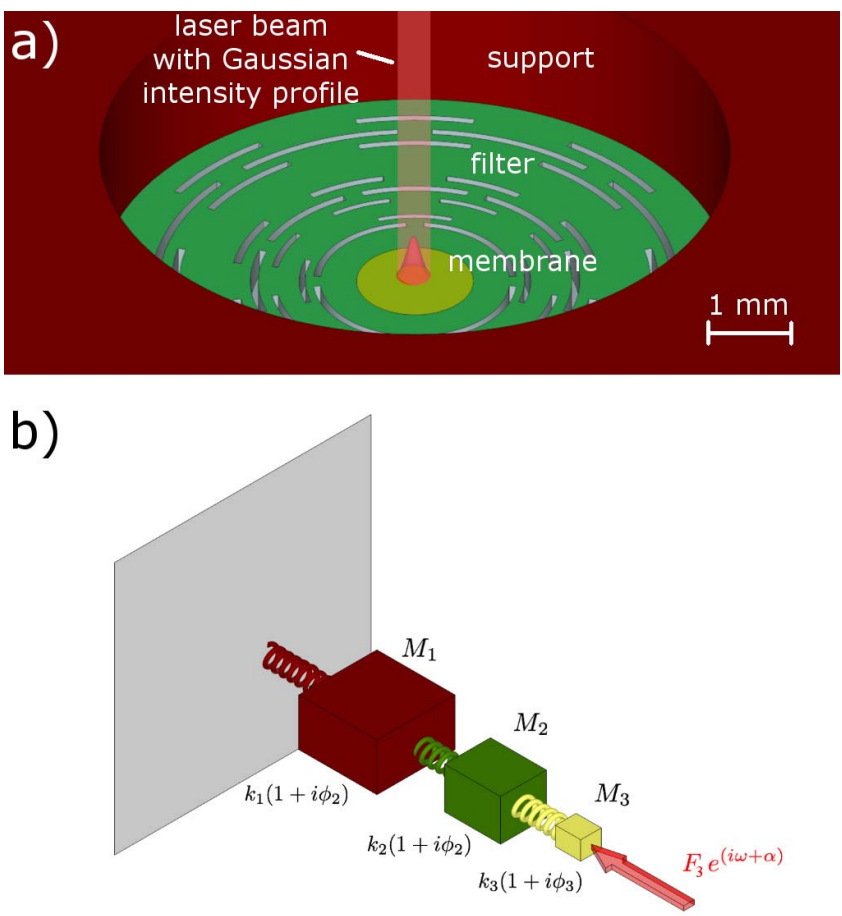

Fig. 2. (a) Model used for in the FEM analysis for the calculation of the thermal noise and the evaluation of the $Q$-factor. (b) Lumped-model of the three resonators (membrane-loss shield-wafer) employed for the physical interpretation of the recoil losses, for the evaluation of the thermal noise at the membrane surface and for the estimation of the $Q$-factor. The impinging radiation force on mass $M_{3}$ is also shown.

low frequency displacement noise. This approach will make the $Q$-factor independent from the internal resonant modes of the system setup. In contrast, commercial TMAH/KOH wet-etched SiN membranes have a $Q$-factor dependent on the specific clamping implemented in the setup.

Given that a dissipative system does not admit a rigorous description in terms of normal modes [24], we evaluate by a numerical analysis the mechanical susceptibility and the associated loss of the motion of the membrane as seen from the readout port. In our system the optical readout samples the displacement of the surface on a circular area with a Gaussian intensity profile centered on the membrane, as shown in Fig. 2a. We point out that the results of the analysis are only slightly dependent on the implementation details, and the optimization remains valid if the beam shape is changed or if a radio frequency readout is used.

In Fig. 2 we show a CAD image of the 3D real system, a practical implementation of a loss-shield made of flexural and torsional joints and the corresponding three-mode lumped resonating system. In both cases we indicate the application point of the laser beam used for the detection of the displacement.

As a first step, we calculated the mechanical response of the lumped tree-mode oscillator, driven by a harmonic force. The effective loss (and therefore the quality factor) is evaluated from the imaginary part of the complex susceptibility. The parameters of the model are then optimized to obtain $Q$-factors of the order of $10^{7}$. As a second step, we study the 3D model 
by Finite Element and tune its geometry to match the dynamic admittance of the optimal three-oscillator model.

Referring to Fig. $2 b$, in the frequency domain the displacement array is related to the force array by a $3 \times 3$ mechanical impedance matrix as: $\left[\tilde{Z}_{i j}(\omega)\right] \tilde{\mathbf{u}}=\tilde{\mathbf{F}}_{d}$, where $\tilde{\mathbf{u}}=$ $\left[u_{1}, u_{2}, u_{3}\right]^{T}$ is the displacement vector and $\tilde{\mathbf{F}}_{d}$ is the driving force vector. The tridiagonal matrix $\left[\tilde{Z}_{i j}(\omega)\right]$ can be easily obtained as a function of the complex spring constants $\hat{k}_{n}=$ $k_{n}\left(1+i \phi_{n}\right)$ (with $k_{n}$ the spring constant and $\phi_{n}$ the associated loss factor):

$$
\begin{aligned}
& {\left[\tilde{Z}_{i j}(\omega)\right]} \\
& =\left(\begin{array}{ccc}
-M_{1} \omega^{2}+\hat{k}_{1} & -\hat{k}_{1} & 0 \\
-\hat{k}_{1} & -M_{2} \omega^{2}+\hat{k}_{1}+\hat{k}_{2} & -\hat{k}_{2} \\
0 & -\hat{k}_{2} & -M_{3} \omega^{2}+\hat{k}_{2}+\hat{k}_{3}
\end{array}\right)
\end{aligned}
$$

Here $\left(M_{1}, \hat{k}_{1}\right)$ are the effective mass and the complex spring constant of the support (wafer + OFHC copper support), $\left(M_{2}, \hat{k}_{2}\right)$ are the effective mass and the complex spring constant of the loss-shield structure and $\left(M_{3}, \hat{k}_{3}\right)$ are the effective mass and the complex spring constant of the membrane. The formal solution of the dynamical equation is $\tilde{\mathbf{u}}=\left[\tilde{Y}_{i j}(\omega)\right] \tilde{\mathbf{F}}_{d}$, where $\left[\tilde{Y}_{i j}(\omega)\right]=\left[\tilde{Z}_{i j}(\omega)\right]^{-1}$ is the admittance matrix of the system. This equation could be solved to evaluate the resonant frequencies and the complex normal modes of the system, but on a practical level we need the response of the membrane $M_{3}$ to a single external force $F_{3}$, as the membrane's position is the only measured variable in the system. In agreement with the fluctuation-dissipation theorem, this is equivalent to the study of the thermal noise power spectrum of the oscillator, as seen from the readout port [25]:

$$
S(\omega)=-\frac{4 k_{B} T}{\omega} \Im\left\{\left[\tilde{Y}_{i j}(\omega)\right]\left[0,0, \tilde{F}_{3}\right]^{T}\right\} .
$$

This curve has a peak at the resonant frequency of the membrane oscillator, and the full width at half maximum of this peak gives a measurement of the quality factor. We point out that this approach estimates the quality factor measured by the dynamical response of the "coupled membrane" (i.e. the membrane coupled to its support system), that is the experimentally accessible quantity. Given that the membrane is only weakly coupled with its support, its admittance will remain similar in shape to the uncoupled oscillator case:

$$
\Im\left(\tilde{Y}^{c}(\omega)\right) \simeq-\frac{1}{\bar{M}_{m}} \frac{\omega_{m}^{2} \bar{\phi}_{m}}{\left(\omega^{2}-\bar{\omega}_{m}^{2}\right)^{2}+\bar{\phi}_{m}^{4} \bar{\omega}_{m}^{4}} .
$$

with some proper values of effective mass $\bar{M}_{m}$, effective frequency $\bar{\omega}_{m}$ and effective loss $\bar{\phi}_{m}$. These effective values depend on the geometrical parameters of the loss shield and wafer, $\left(M_{1}, \hat{k}_{1}\right)$ and $\left(M_{2}, \hat{k}_{2}\right)$. Numerical evaluation show that the corrections to the membrane mass and resonant frequency are negligible $\left(\bar{M}_{m} \simeq M_{3}\right.$ and $\left.\bar{\omega}_{m} \simeq \omega_{3}\right)$, while the quality factor $\bar{Q}_{m}=\phi_{m}^{-1}$ can become smaller than the uncoupled $Q_{3}$ if the frequency $\omega_{1}$ of the wafer approaches $\omega_{m}$. In this case the loss shield turns out to be very effective in protecting the quality factor of the membrane from excessive reduction.
TABLE I

PARAMETERS OF THE UNCOUPLED OSCILLATORS USED IN THE 3-OSCILLATOR MODEL

\begin{tabular}{|c|c|}
\hline & 3-oscillator model \\
\hline$M_{1}$ & $5 \times 10^{-5} \mathrm{Kg}$ \\
$\omega_{1} / 2 \pi$ & $227 \mathrm{kHz}$ \\
$k_{1}=M_{1} \omega_{1}^{2}$ & $1.01 \times 10^{8} \mathrm{~N} / \mathrm{m}$ \\
$\phi_{1}$ & $10^{-3}$ \\
$M_{2}$ & $1 \times 10^{-5} \mathrm{Kg}$ \\
$\omega_{2} / 2 \pi$ & $30 \mathrm{kHz}$ \\
$k_{2}=M_{2} \omega_{2}^{2}$ & $3.55 \times 10^{5} \mathrm{~N} / \mathrm{m}$ \\
$\phi_{2}$ & $10^{-3}$ \\
$M_{3}$ & $1.5 \times 10^{-10} \mathrm{Kg}$ \\
$\omega_{3} / 2 \pi$ & $230 \mathrm{kHz}$ \\
$k_{3}=M_{3} \omega_{3}^{2}$ & $3.13 \times 10^{2} \mathrm{~N} / \mathrm{m}$ \\
$\phi_{3}$ & $10^{-7}$ \\
\hline
\end{tabular}

The optimal parameters of the loss shield, shown in Table I have been found with a numerical approach based on the model of Fig. 2b [19]. The actual geometry consistent with these parameters has been developed with the help of FEM modeling.

\section{Intrinsic Losses}

A critical issue concerning the design of MOMS oscillator is the thin oxide layer that is used as etch-stop layer for the Deep-RIE on the back side. The thin TEOS $\mathrm{SiO}_{2}$ layer is shown in the design concept of the oscillator Figure 1. This layer is completely removed from the membrane in a release step following the Deep-Rie etching of the substrate. This layer, if not removed properly at the membrane's edge, can originate an additional loss depending on its thickness. In particular, the dissipation behaviors of this layer can be critical at cryogenic temperatures, commonly required in quantum optomechanics, as $\mathrm{SiO}_{2}$ has a dissipation peak at $50 \mathrm{~K}$ [26] that affects its behavior down to Liquid Helium temperatures.

The intrinsic quality factor $\left(Q_{\text {int }}=\phi_{\text {int }}^{-1}\right)$ of a free-standing membrane dominated by intrinsic stress $\sigma_{0}$ can be calculated, for a square-shaped membrane, with the approach described in [21]:

$$
Q_{\text {int }}\left(n_{1}, n_{2}, \sigma_{0}\right)=\frac{1}{\lambda} \frac{E^{\mathrm{Re}}}{E^{\mathrm{Im}}}\left(1+\lambda \frac{n_{1}^{2}+n_{2}^{2}}{4}\right)^{-1}
$$

where $\left(n_{1}, n_{2}\right)$ are the mode indexes, $\lambda=\sqrt{2 F / \sigma_{0} h l^{2}}$ with $F$ the flexural rigidity, $h, l$ the thickness and the side length of the square-shaped $\mathrm{SiN}$ layer. $E^{\mathrm{Re}}$ and $E^{\mathrm{Im}}$ are the real and imaginary parts of the complex Young's modulus, that are related to the loss factor according to the following relation: $\hat{E}=E^{\mathrm{Re}}+i E^{\mathrm{Im}} \equiv E(1+i \phi)$. We take 7 as an estimate for the quality factor in the case of a circular membrane with similar frequency.

In 7 , the physical meaning of $\lambda$ is the ratio between the bending energy to the elongation energy for the fundamental mode (in our case $\lambda=1.08 \times 10^{-3}$ ). The loss at the edge is described by the term independent from the mode indexes 
TABLE II

Material Parameters USED For EVAluating Dissipation OF THE $\mathrm{SiO}_{2}$ LAYER AT LOW AND ROOM TEMPERATURES (CL - Clamping Limit - TL Thermoelastic Limit)

\begin{tabular}{|c|c||c|}
\hline & $\begin{array}{c}\text { Low temperature } \\
\text { (at LHe) }\end{array}$ & $\begin{array}{c}\text { Room temperature } \\
\text { (at RT) }\end{array}$ \\
\hline \hline$E_{\mathrm{SiO}_{2}}(\mathrm{GPa})$ & 77 & 77 \\
$E_{\mathrm{SiN}}(\mathrm{GPa})$ & 260 & 260 \\
$\rho_{\mathrm{SiO}_{2}}\left(\mathrm{Kg} / \mathrm{m}^{3}\right)$ & 2800 & 2800 \\
$\rho_{\mathrm{SiN}}\left(\mathrm{Kg} / \mathrm{m}^{3}\right)$ & 2100 & 2100 \\
$\sigma_{0}(\mathrm{MPa})$ & 800 & 800 \\
$\phi_{\mathrm{SiO}}$ & $1 \times 10^{-3}$ & $5 \times 10^{-4}$ \\
$\phi_{\mathrm{SiN}}$ & $2 \times 10^{-5}$ & $2 \times 10^{-5}$ \\
$\phi_{\mathrm{Si}}$ & $1 \times 10^{-6}$ & $3 \times 10^{-4}(\mathrm{TL})$ \\
$\phi_{\text {wafer }}$ & $1 \times 10^{-3}(\mathrm{CL})$ & $1 \times 10^{-3}(\mathrm{CL})$ \\
\hline
\end{tabular}

$\left(n_{1}, n_{2}\right)$, that is:

$$
Q_{\text {edge }}\left(\sigma_{0}\right)=\frac{1}{\lambda} \frac{E^{\mathrm{Re}}}{E^{\mathrm{Im}}}
$$

To evaluate the lower limit of the quality factor due to the TEOS oxide layer, we estimate the components of the complex Young modulus by considering the effective thin film layer:

$$
\begin{aligned}
E^{\mathrm{Re}} & =E_{\mathrm{SiN}} f_{v_{\mathrm{SiN}}}+E_{\mathrm{SiO}_{2}} f_{v_{\mathrm{SiO}_{2}}} \\
& =\frac{E_{\mathrm{SiN}} h_{\mathrm{SiN}}+E_{\mathrm{SiO}_{2}} h_{\mathrm{SiO}_{2}}}{h_{\mathrm{SiN}}+h_{\mathrm{SiO}_{2}}} \\
E^{I m} & =E_{\mathrm{SiN}} \phi_{\mathrm{SiN}} f_{v_{\mathrm{SiN}}}+E_{\mathrm{SiO}_{2}} \phi_{\mathrm{SiO}_{2}} f_{v_{\mathrm{SiO}_{2}}} \\
& =\frac{E_{\mathrm{SiN}} \phi_{\mathrm{SiN}} h_{\mathrm{SiN}}+E_{\mathrm{SiO}_{2}} \phi_{\mathrm{SiO}_{2}} h_{\mathrm{SiO}_{2}}}{h_{\mathrm{SiN}}+h_{\mathrm{SiO}_{2}}}
\end{aligned}
$$

where $E^{\mathrm{Re}}$ and $E^{\mathrm{Im}}$ are the effective real and imaginary Young modulus, $\mathrm{f}_{\mathrm{v}_{\mathrm{SiN}}}, \mathrm{f}_{\mathrm{v}_{\mathrm{SiO}_{2}}}$ are the volume fractions while $E_{\mathrm{SiN}}, E_{\mathrm{SiO}_{2}}$ are the Young modulus and the loss angle $\phi_{\mathrm{SiN}}, \phi_{\mathrm{SiO}_{2}}$, as reported in Table II.

These data are used to simulate the total membrane loss by FEM, showing that for a system without oxide the Q-factor can potentially reach $5 \times 10^{7}$ at $4 \mathrm{~K}$, while a reduction of the overall Q-factor is expected in the bilayer system $\mathrm{SiN} / \mathrm{SiO}_{2}$ $(100 / 290) \mathrm{nm}$. At room temperature the extra dissipation due to the $\mathrm{SiO}_{2}$ layer is negligible. Results at $4 \mathrm{~K}$ and $300 \mathrm{~K}$ are reported in Figure 3.

\section{MICROFABRICATION}

Resonators were fabricated exploiting MEMS bulkmicromachining by Deep-Reaction Ion Etching (DRIE) and through wafer two-side processing, a process which has already demonstrated the capability of producing low-loss micro-mechanical systems [27], [28]. Resonators were fabricated from 4-inches Silicon-On-Insulator (SOI) Floating Zone wafer (Icemos Technology Ltd.) of total thickness $1000 \pm$ $5 \mu \mathrm{m}$ (device layer $250 \mu \mathrm{m}$ ), orientation $<100>$ with resistivity $>1 \mathrm{k} \Omega \mathrm{cm}$ (Fig. 4 step 1 ). We patterned the handle and device layers by multiple DRIE etching steps, using the $2 \mu \mathrm{m}$ buried oxide as etch stop layer.

Firstly, on the device layer we deposited a multilayer thin-film stack composed of a low pressure chemical vapor

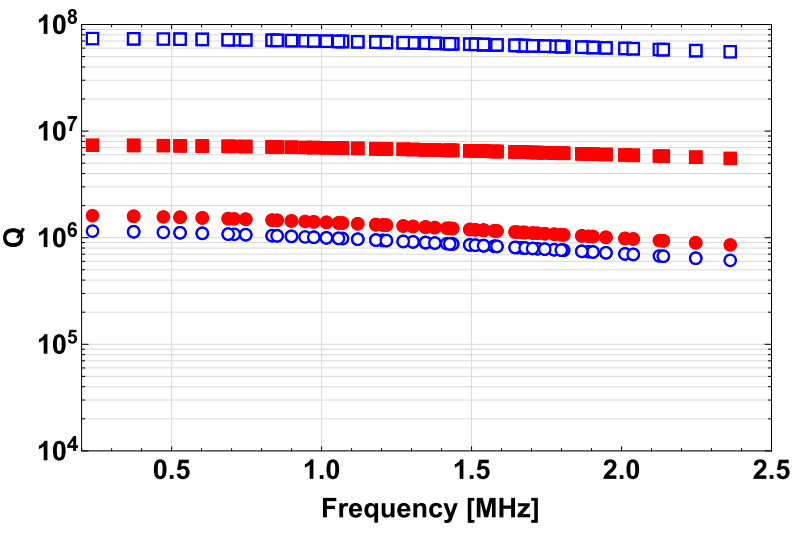

Fig. 3. Results of FEM simulations. Q-factor at room temperature (300 K): (red-filled squares) $100 \mathrm{~nm}$ thick SiN membrane; (red-filled circles) bilayer $\mathrm{SiN} / \mathrm{SiO}_{2}(100 / 290) \mathrm{nm}$. Q-factor at Liquid Helium (4 K): (blue squares) $100 \mathrm{~nm}$ thick $\mathrm{SiN}$ membrane; (blue circles) bilayer $\mathrm{SiN} / \mathrm{SiO}_{2}$ $(100 / 290) \mathrm{nm}$

deposition (LPCVD) tetraethyl orthosilicate (TEOS) oxide film with thickness $290 \mathrm{~nm}$ (on the bottom), the 100-nm-thick LPCVD SiN membrane (in the middle) and a $1 \mu \mathrm{m}$ thick RF-sputtered pure Al layer (on the top) as it is shown in Fig. 4 step 2. The TEOS oxide works as etch stop layer during DRIE etching steps while the $\mathrm{Al}$ layer is used as front side mask and as protection layer for the wet/dry etching processing phases. Keeping the deposition temperature fixed at $800^{\circ} \mathrm{C}$, a SiN layer with a residual stress of $0.8 \mathrm{GPa}$ was obtained by tuning the flow of the gas precursors (the resulting stress is measured on a Si wafer by curvature methods TENCOR Flexus FLX-2908). Before RF-sputtering of the $\mathrm{Al}$ layer, a plasma enhanced chemical vapor deposition (PECVD) oxide mask of thickness $6 \mu \mathrm{m}$ was deposited on the back side at $400^{\circ} \mathrm{C}$ (Fig. 4 step 2). Silicon etching has been realized by means of DRIE two pulse BOSCH process in Omega i2L Rapier at $20{ }^{\circ} \mathrm{C}$. A multi-level etching processing was performed to obtain the membrane's hole and the loss-shield masses, using resist AZ9260 and the nested PECVD oxide mask. With reference to Fig. 4 step 3-4 we first etch $200 \mu \mathrm{m}$ and after stripping the resist we performed a second etching step of $550 \mu \mathrm{m}$ landing on the buried-oxide.

The estimated average etching rates were about $1.38 \mu \mathrm{m} /$ cycle for $\mathrm{Si}$ and $5 \mathrm{~nm} /$ cycle for the PECVD oxide. The gas-flow rate and number of cycles were optimized to control the aspect ratio dependent etching and notching. Afterwards, we removed the buried oxide by means of an HF-based solution (Fig. 4 step 5) that fully etches the PECVD oxide mask. The SiN layer on the back side works as etch stop layer for HF and protects the thermal oxide underneath. To release the thin-film stack we performed the third DRIE etching step $(250 \mu \mathrm{m})$ exploiting the $2 \mu \mathrm{m}$ thermal oxide as mask (Fig. 4 step 6). We divided the etching step in two sub-steps in order to prevent membranes failure. We saw re-entrant sidewalls due to aspect ratio dependent etching rates. By a last DRIE etching step (Fig. 4 step 7) we obtained the front-side suspending structure in the $250 \mu \mathrm{m}$ device layer; in this case we used a $8 \mu \mathrm{m}$ photoresists AZ9260 (to avoid micro masking of the Al layer). Front and back 
(1) SOI wafer

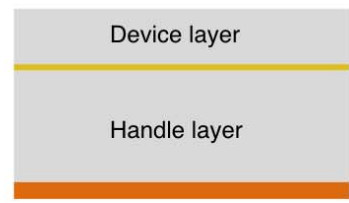

(2) Thin film depositions

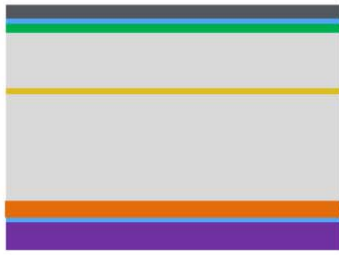

(3) 1st BS deep-RIE

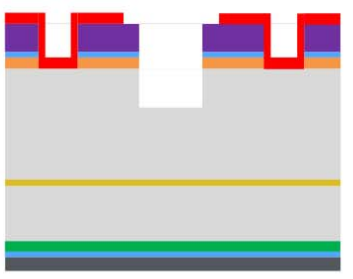

(4) 2nd BS deep-RIE

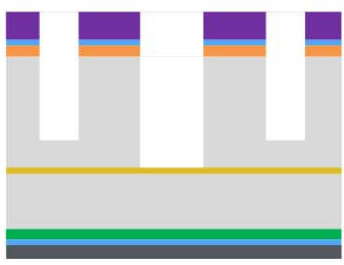

Thermal oxide

Buried oxide

LPCVD SiN

LPCVD Oxide
(5) Box-oxide etching

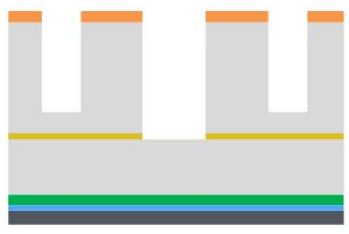

(6) 3rd BS deep-RIE

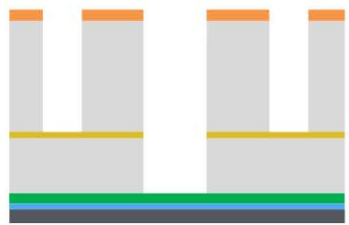

(7) 4th FS deep-RIE

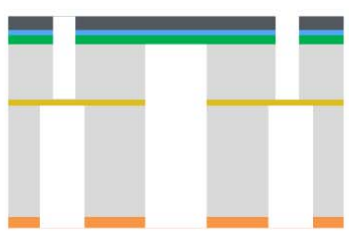

(8) membrane release

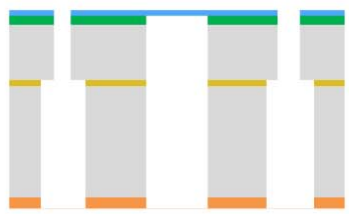

PECVD Oxide

Al layer

Photoresist
Thermal oxide

Buried oxide

LPCVD SiN

LPCVD Oxide

PECVD Oxide

Al layer

Photoresist

BS: BACK-SIDE

FS: FRONT-SIDE

deep-RIE: deep - REACTIVE ION

ETCHING for Silicon (low temperature Bosch process)

Fig. 4. (1) SOI wafer; (2) thin film depositions: LPCVD SiN, PECVD $\mathrm{SiO}_{2}, \mathrm{Al}$; (3) $1^{\mathrm{st}}$ DRIE etching on the back-side (BS) with a resist mask with a nested oxide mask; (4) photoresist removal and $2^{\text {nd }}$ back-side (BS) etching using the nested oxide mask; (5) HF wet etching of the buried oxide; (6) $3^{\text {nd }}$ back-side (BS) etching; (7) front-side (FS) resist AZ9260 and Al patterning followed by $4^{\text {th }}$ front-side (FS) DRIE etching; (8) membrane release: Al stripping by PES chemical bath and oxide HF wet etching.

side of the whole oscillator structure are shown in Fig. 5 and Fig. 6 respectively, detailed views of the torsional-flexural joints are shown in Fig. 7 and Fig. 8.

The last step is the SiN membrane release. First we stripped the Al layer using a PES solution, then by means of an HF-based solution we etched the TEOS oxide (Fig. 4 step 8). A HF over-etch is needed in this phase in order to completely release the SiN layer, as any remnant TEOS oxide layer could spoil the mechanical Q-factor of the membrane. The appearance of the undercut as it is shown in Fig. 9 (b) represents a good way to set a proper over-etching time. The process ends with dicing and cleaning from resist residues by oxygen plasma (TEPLA) and by $\mathrm{HNO}_{3}$ solution $99 \%$.

\section{EXPERIMENTAL CHARACTERIZATION}

The experimental set-up used for the measurement of the quality factor is shown in Fig. 10. A beam of $3 \mathrm{~mW}$ reflected by the PBS1 from our light source, a Nd:YAG at $1064 \mathrm{~nm}$, is aligned in a Michelson interferometer followed by a balanced homodyne detection. In details, a polarizing beam-splitter (PBS2) divides the beam into two parts, orthogonally polarized, forming the Michelson interferometer arms. At the end of the first one (reference arm) an electromagnetically-driven mirror M1 is used for phase-locking the interferometer in the condition of maximum displacement sensitivity. A double pass through a quarter-wave plate rotates by $90^{\circ}$ the polarization of this beam, which is 


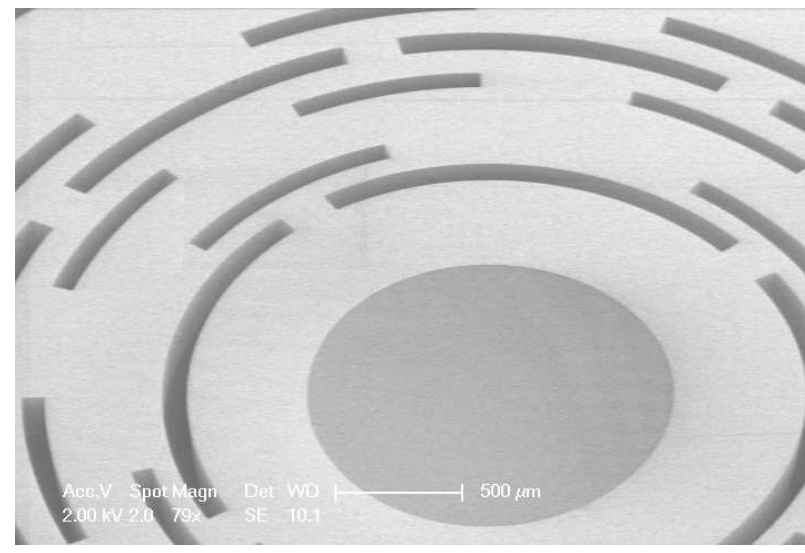

Fig. 5. SEM image of the front side of the circular SiN membrane oscillator with the loss-shield.

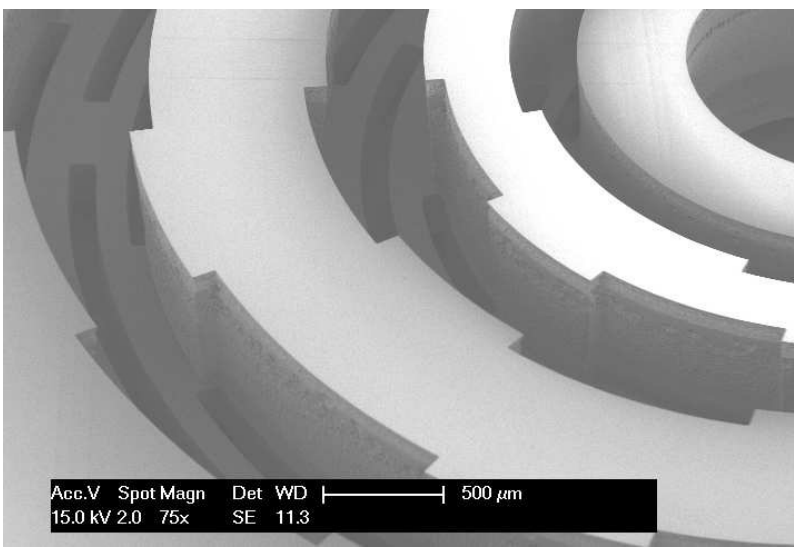

Fig. 6. SEM image of the back side of the membrane oscillator showing the loss-shield masses.

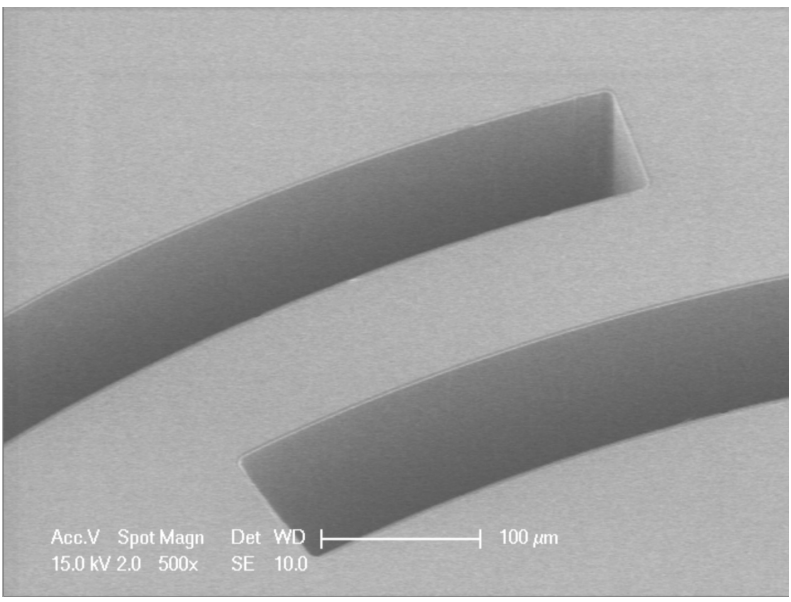

Fig. 7. SEM image of the front side showing the detail of the torsional-flexural joints.

then transmitted by PBS2. The second beam (sensing arm) is focused on the membrane oscillator firmly fixed inside the vacuum chamber, and after reflection and double pass through the quarter-wave plate is reflected by the PBS2, where it overlaps with the reference beam reflected by M1. The overlapped beams are then monitored by a homodyne detection, consisting

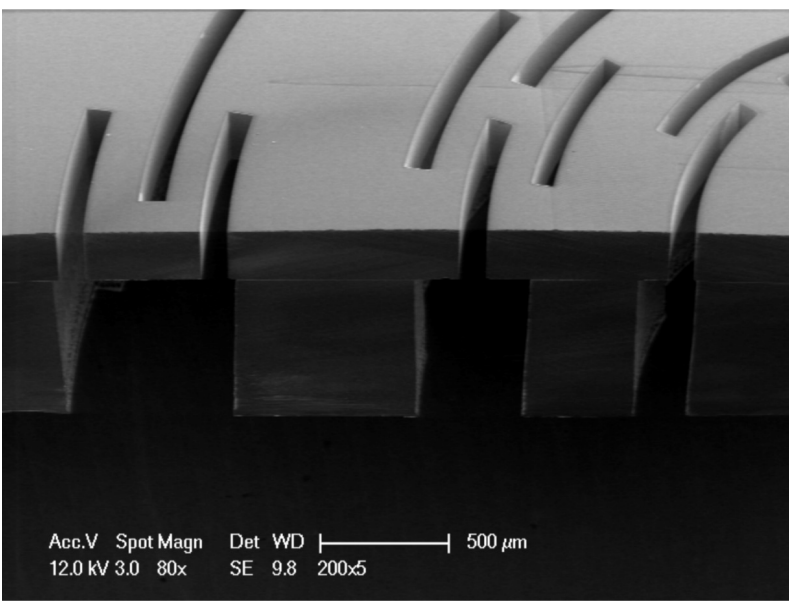

Fig. 8. SEM image of the cross-section of the device showing torsional-flexural joints of the shield-loss stage.

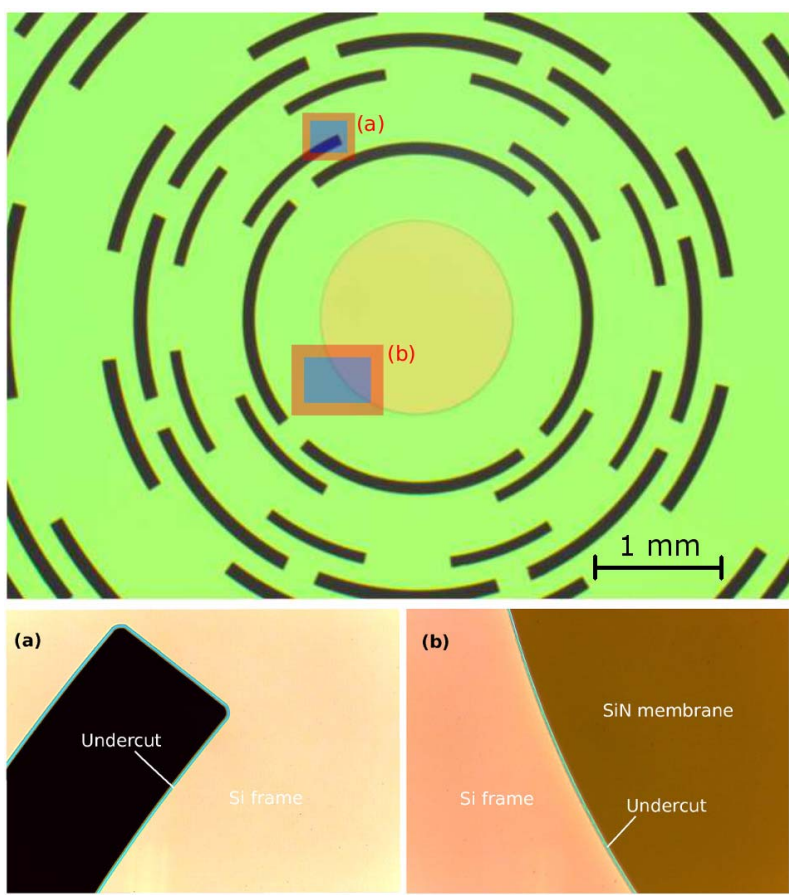

Fig. 9. (Top) Optical image of the front side of the SiN circular membrane with its integrated loss-shield. (Bottom) Detailed view of the (a) and (b) areas showing the undercut of $\mathrm{SiO}_{2}$ by $\mathrm{HF}$ wet etching for the membrane release.

of a half-wave plate, rotating the polarizations by $45^{\circ}$, and a polarizing beamsplitter (PBS3) that divides the radiation into two equal parts sent to the photodiodes PD1 and PD2, whose outputs are subtracted. The signal obtained is a null-average, sinusoidal function of the path difference in the interferometer. Such a scheme is barely sensitive to laser power fluctuations. The difference signal is used as error signal in the locking servo-loop (the locking bandwidth is about $1 \mathrm{kHz}$ ) and also sent to the acquisition instruments for sensing the displacement of the membrane. The quality factor of the resonant modes are evaluated by the measurement of the free decay time after resonant excitation with a piezoelectric crystal. When the drive signal is removed, the mechanical vibration follows 


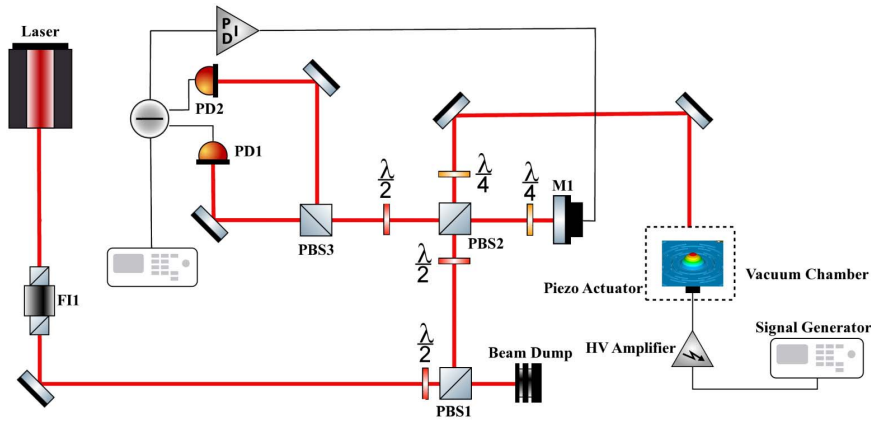

Fig. 10. Optical scheme of the Michelson interferometer apparatus for the measurement of the mechanical Q-factor of the modes of the SiN membrane. The main beam is split in PBS2 (polarizing beam-splitter) and then recombines in PBS2-PBS3 after reflection from mirror $M_{1}$ (reference arm) and the SiN membrane (sensing arm).
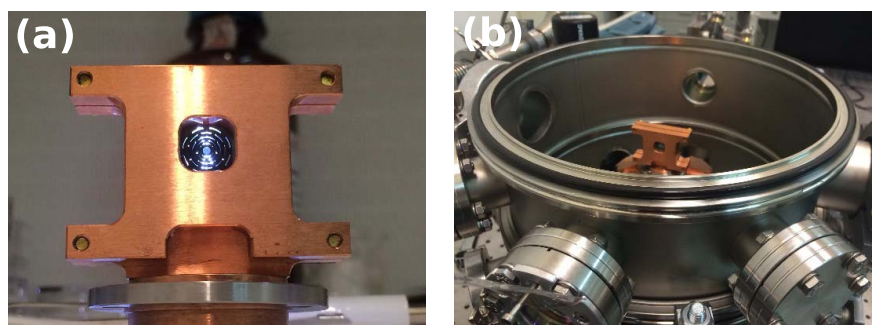

Fig. 11. (a) Clamping frame made of OFHC copper with the oscillator housed in the middle; (b) the system is mounted on top of a seismic filter and housed inside a vacuum chamber.

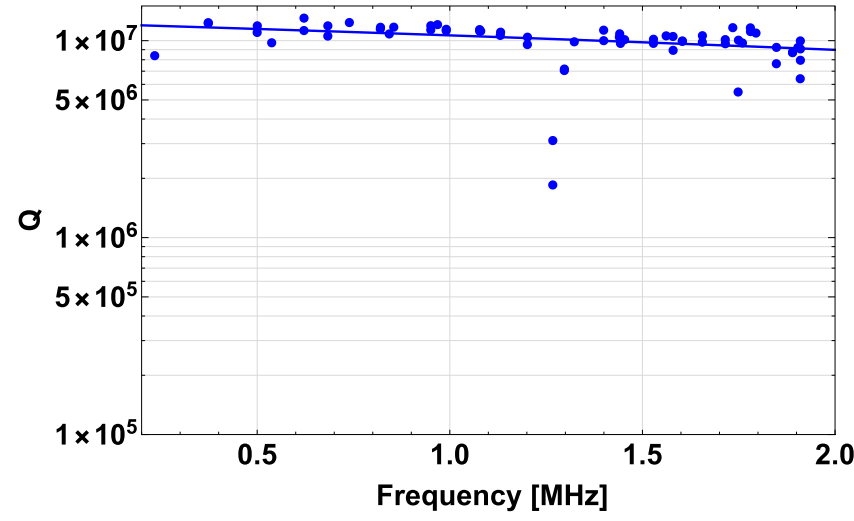

Fig. 12. The Q-factor at room temperature of the resonant modes of a reference device (wf1726-14). We show as a continuous line the least-square regression line of these data, that is in good agreement with the results expected for a square-shaped membrane of the same size [21].

an exponentially damped decay whose envelope amplitude varies according to: $u(t)=u_{0} \exp \left(-t / \tau_{n_{0} n_{1}}\right)$ where $\tau_{n m}=$ $Q / \pi v_{n_{0} n_{1}}$ is the decay time of the mode and $v_{n_{0} n_{1}}$ is the resonance frequency of mode with indexes $\left(n_{0}, n_{1}\right)$, as explained in the next Section. In Fig. 11(a) we show the customized clamping systems for a $14 \times 14 \mathrm{~mm}^{2}$ chip and the vacuum chamber Fig. 11(b). The clamping system is isolated from vibrations with a spring-mass system linked to the optical table from the bottom and the pressure in the vacuum chamber is about $1 \times 10^{-6}$ mbar to prevent gas damping effects.

\section{RESUlts AND Discussion}

In Fig. 12 we report the quality factor of all of the frequency modes (up to $2 \mathrm{MHz}$ ) of a reference device (wf1726-14).

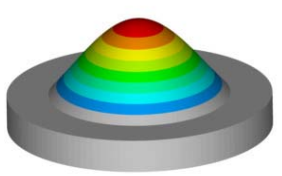

$\left(\begin{array}{ll}0 & 1\end{array}\right)$

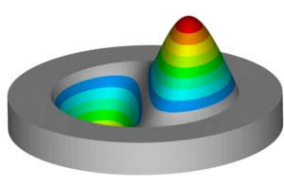

(1 1)

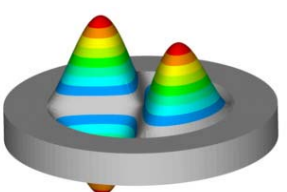

(2 1$)$
Fig. 13. Modal shapes the circular membrane with mode indexes $\left(n_{0}, n_{1}\right)$. The color scale, from light gray to red, is proportional to the absolute displacement from the equilibrium position.

TABLE III

Ellipsometry MEASUREMENT OF THE THICKNESS OF THE TEOS OXIDE AND THE SiN And the AVERAge Undercut After HF ETCHING

\begin{tabular}{cccc} 
DEVICE & $\begin{array}{c}\mathrm{t}_{\mathrm{ox}} \pm 0.5 \% \\
{[\mathrm{~nm}]}\end{array}$ & $\begin{array}{c}\mathrm{t}_{\mathrm{SiN}} \pm 0.5 \% \\
{[\mathrm{~nm}]}\end{array}$ & $\begin{array}{c}\mathrm{u} \pm 5 \% \\
{[\mu m]}\end{array}$ \\
\hline \hline w1722-15 & 298 & 108 & 5.48 \\
$\mathrm{w} 1726-14$ & 298 & 100 & 2.35 \\
$\mathrm{w} 1733-13$ & 302 & 109 & 6.37 \\
w1736-12 & 302 & 109 & 2.60 \\
w1752-14 & 298 & 100 & 2.21 \\
\hline \hline
\end{tabular}

In comparison with commercial devices, measured for instance in [21], these results highlight the effectiveness of the integrated loss-shield, as almost all modes achieve a high $Q$ value, in good agreement with what is expected by (7) for squareshaped membranes.

In general, microfabricated systems should ensure good reproducibility of the operating parameters. To evaluate the robustness of our microfabrication process we compare the $Q$-factor measurements for five devices obtained in five different runs. Relevant geometrical parameters related to the thin film oxide and nitride layers are reported in Table III. In Table IV we report the intrinsic stress $\sigma_{0}$, the measured diameter D and the $Q$-factor measurements for three vibrational modes. From these data we estimate the sensitivity of the $Q$-factor to the variations of the intrinsic stress $\Delta \sigma_{0}$ and undercut $\Delta U_{o x}$. The analysis refer to the three low-frequency oscillations with mode indexes $\left(n_{0}, n_{1}\right)=(01) /(11) /(21)$, with modal shape shown in Figure 13. We point out that for these low frequencies modes a high quality factor can be obtained only thanks to the loss-shield stage.

Stress variation are originated by pressure fluctuations and small variations of the gas ratio $\mathrm{NH}_{3} / \mathrm{SiH}_{4}$ during the LPCVD deposition. A precise estimate of the intrinsic stress $\sigma_{0}$ of the membrane, reported in Table IV, was derived from the interferometric measurement of the frequency $v_{0}$ (Fig. 10). In fact the stress and the resonant frequency are connected by the relation: $v_{n_{0}, n_{1}}=v_{0} \alpha_{n_{0}, n_{1}}$, where $\alpha_{n_{0}, n_{1}}$ is the $n_{1}$-th root of the Bessel polynomial of order $n_{0}$ and $\nu_{0}=\frac{1}{\pi D_{0}} \sqrt{\frac{\sigma_{0}}{\rho}}$. Here $\rho=2800 \mathrm{Kg} / \mathrm{m}^{3}$ is the density of the $\mathrm{SiN}$ film, and the diameter $\mathrm{D}_{0}$ was directly measured on the device by Leica DM750M optical microscope with magnification of $50 \times$. According to 7, the mechanical $Q$-factor in a square membrane is directly related to the intrinsic stress $\sigma_{0}$ by the dilution effect of the mechanical losses in SiN films. For this reason it is not surprising that higher quality factors are generally observed 
TABLE IV

Experimental Measurements of Device Parameters and Mechanical Quality Factor at RoOM TEMPERATURE $(300 \mathrm{~K})$

\begin{tabular}{|c|c|c|c|c|c|c|}
\hline DEVICE & $\begin{array}{c}\sigma_{0} \\
{[\mathrm{MPa}]}\end{array}$ & $\begin{array}{c}D \\
{[\mathrm{~mm}]}\end{array}$ & $\begin{array}{c}\nu_{\mathrm{mn}} \\
{[\mathrm{kHz}]}\end{array}$ & $\begin{array}{c}\mathrm{Q} \\
{\left[10^{6}\right]}\end{array}$ & $n_{0} n_{1}$ & $\begin{array}{l}\mathrm{f} \times \mathrm{Q} \\
{[\mathrm{THz}]}\end{array}$ \\
\hline \multirow{3}{*}{ w1722-15 } & & & 247.47 & 8.5 & (01) & 2.09 \\
\hline & 703.07 & 1.55 & 374.38 & 8.4 & (11) & 3.14 \\
\hline & & & 568.29 & 7.8 & (21) & 4.42 \\
\hline \multirow{3}{*}{ w1726-14 } & & & 233.98 & 8.4 & (01) & 1.96 \\
\hline & 712.22 & 1.65 & 372.96 & 12.5 & (11) & 4.65 \\
\hline & & & 499.95 & 11.1 & $(21)$ & 5.56 \\
\hline \multirow{3}{*}{ w1733-13 } & & & 274.16 & 11.7 & (01) & 3.22 \\
\hline & 862.90 & 1.55 & 438.82 & 14.3 & (11) & 6.27 \\
\hline & & & 718.95 & 9.8 & (21) & 7.06 \\
\hline \multirow{3}{*}{ w1736-12 } & & & 275.91 & 10.9 & (01) & 3.00 \\
\hline & 818.48 & 1.50 & 439.49 & 11.1 & (11) & 4.87 \\
\hline & & & 589.91 & 10.6 & (21) & 6.25 \\
\hline \multirow{3}{*}{ w1752-14 } & & & 241.82 & 8.7 & (01) & 2.09 \\
\hline & 671.33 & 1.55 & 384.88 & 9.1 & (11) & 3.49 \\
\hline & & & 516.77 & 8.7 & (21) & 4.50 \\
\hline
\end{tabular}

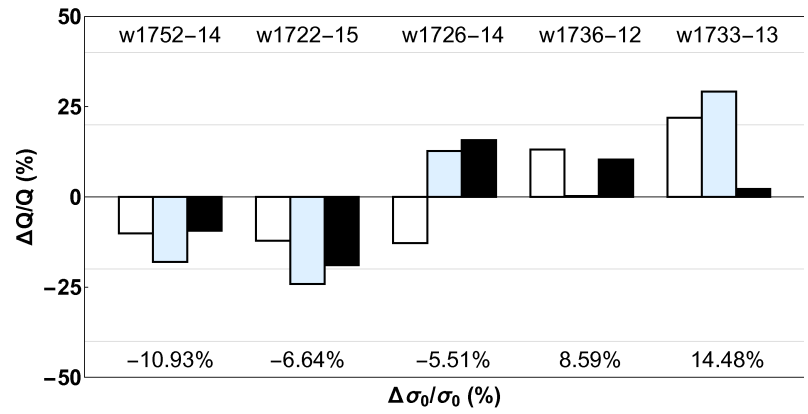

Fig. 14. Sensitivity of the relative variations of the $Q$-factor, $\Delta Q / Q$, with respect to the relative variation of the stress $\Delta \sigma_{0} / \sigma_{0}$ for the first three modes: (white) mode indexes (01); (light-blue) mode indexes (11); (black) mode indexes (21). The reference values are the average of the measurements over the 5 devices: $753 \mathrm{MPa}$ for the stress and $\left(9.6 \times 10^{6}, 11.06 \times 10^{6}, 9.60 \times 10^{6}\right)$ for quality factors of the three modes.

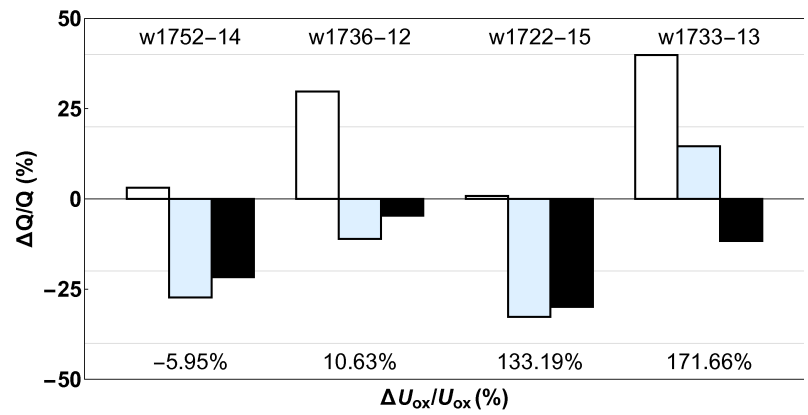

Fig. 15. Sensitivity of the relative variations of the $Q$-factor, $\Delta Q / Q$, with respect to the relative variation of the undercut $\Delta U_{o x} / U_{o x}$ for the first three modes: (white) mode indexes (01); (light-blue) mode indexes (11); (black) mode indexes (21). The relative variations are deduced from the values measured in the reference device w1726-14.

when the stress is higher. In Fig. 14 we show the relative variation of $\Delta Q / Q$ as a function of the relative variation of stress, taking as reference value the average of the measured stress over the five different devices.
The undercut $\mathrm{U}_{\mathrm{ox}}$ is roughly proportional to the etching time chosen for the release of the SiN membrane (Fig. 4 step 8). Actually it is used to check if the SiN membrane is fully released from the $\mathrm{SiO} 2$ layer underneath: no undercut means no free-standing membrane. Starting from the reference process, corresponding to device w1726-14, different etching time were tested to check possible effects on the quality factor. For each wafer the undercut, measured with Leica DM750M using a magnification of $50 \times$, is reported in Table III. As shown in Fig. 15, where no clear correlation can be seen, the undercut does not influence the $Q$-factor of the modes. Here the relative variations are evaluated from the reference device.

\section{CONCLUSION}

This work presents the microfabrication process for the production of high-Q SiN membranes with an integrated on-chip 3D loss-shield. Within its operating bandwidth $(0-5 \mathrm{MHz})$, the device preserves the intrinsic Q-factor of the pretension round-shaped nanomembrane, regardless of the modal order or resonant frequency. In particular, $Q$-factors at room temperature are about $10^{7}$, achieving the minimum requirement for room-temperature quantum optomechanics $Q \times f>6.2 \mathrm{THz}$ for all modes with frequency higher than $600 \mathrm{kHz}$.

The device is obtained by MEMS bulk-micromachining via Deep-Reaction Ion Etching (DRIE), combined with a two-side processing on a thick SOI wafer. The microfabrication process is quite flexible and additional layers could be deposited over the SiN membrane before the DRIE steps, if required for a sensing application. Therefore, such oscillator is a promising candidate for quantum sensing applications in the context of the emerging field of Quantum Technologies. Additional layers can be deposited onto the SiN layer before fabrication, for sensing applications or to tailor the membrane reflectivity, without increasing the process complexity.

\section{ACKNOWLEDGMENT}

The authors would like to thank the EKL IC Process Group for their technological support.

\section{REFERENCES}

[1] I. Favero and K. Karrai, "Optomechanics of deformable optical cavities," Nature Photon., vol. 3, pp. 201-205, Apr. 2009, doi: 10.1038/nphoton.2009.42.

[2] M. Aspelmeyer, T. J. Kippenberg, and F. Marquardt, "Cavity optomechanics," Rev. Mod. Phys., vol. 86, no. 4, p. 1391, 2014, doi: 10.1103/ RevModPhys.86.1391.

[3] M. Metcalfe, "Applications of cavity optomechanics," Appl. Phys. Rev., vol. 1, no. 3, p. 031105 , 2014, doi: 10.1063/1.4896029.

[4] A. Pontin et al., "Quantum nondemolition measurement of optical field fluctuations by optomechanical interaction," Phys. Rev. A, Gen. Phys., vol. 97, no. 3, p. $033833,2018$.

[5] I. Pikovski, M. R. Vanner, M. Aspelmeyer, M. S. Kim, and Č. Brukner, "Probing planck-scale physics with quantum optics," Nature Phys., vol. 8, pp. 393-397, Mar. 2012, doi: 10.1038/nphys2262.

[6] M. Bawaj et al., "Probing deformed commutators with macroscopic harmonic oscillators," Nature Commun., vol. 6, Jun. 2015, Art. no. 7503, doi: $10.1038 /$ ncomms 8503 
[7] J. D. Thompson, B. M. Zwickl, A. M. Jayich, F. Marquardt, S. M. Girvin, and J. G. E. Harris, "Strong dispersive coupling of a high-finesse cavity to a micromechanical membrane," Nature, vol. 452, pp. 72-75, Mar. 2008, doi: 10.1038/nature06715.

[8] Y. Tsaturyan, A. Barg, E. S. Polzik, and A. Schliesser, "Ultracoherent nanomechanical resonators via soft clamping and dissipation dilution," Nature Nanotechnol., vol. 12, p. 776-783, Jun. 2017, doi: 10.1038/ nnano.2017.101.

[9] T. P. Purdy, R. W. Peterson, and C. A. Regal, "Observation of radiation pressure shot noise on a macroscopic object," Science, vol. 339, no. 6121, pp. 801-804, 2013, doi: 10.1126/science.1231282.

[10] T. P. Purdy, P. L. Yu, R. W. Peterson, N. S. Kampel, and C. A. Regal, "Strong optomechanical squeezing of light," Phys. Rev. X, vol. 3, no. 3, p. 031012, 2013, doi: 10.1103/PhysRevX.3.031012.

[11] W. H. P. Nielsen, Y. Tsaturyan, C. B. Møller, E. S. Polzik, and A. Schliesser, "Multimode optomechanical system in the quantum regime," Proc. Nat. Acad. Sci. USA, vol. 114, no. 1, pp. 62-66, 2017, doi: 10.1073/pnas.1608412114.

[12] M. Rossi et al., "Enhancing sideband cooling by feedback-controlled light," Phys. Rev. Lett., vol. 119, no. 12, p. 123603, 2017, doi: 10. 1103/PhysRevLett.119.123603.

[13] Q. P. Unterreithmeier, T. Faust, and J. P. Kotthaus, "Damping of nanomechanical resonators," Phys. Rev. Lett., vol. 105, no. 2, p. 027205 , 2010, doi: 10.1103/PhysRevLett.105.027205.

[14] D. J. Wilson, C. A. Regal, S. B. Papp, and H. J. Kimble, "Cavity optomechanics with stoichiometric SiN films," Phys. Rev. Lett., vol. 103, no. 20, p. 207204, 2009, doi: 10.1103/PhysRevLett.103.207204.

[15] R. A. Norte, J. P. Moura, and S. Gröblacher, "Mechanical resonators for quantum optomechanics experiments at room temperature," Phys. Rev. Lett., vol. 116, no. 14, p. 147202, 2014, doi: 10.1103/PhysRevLett.116.147202.

[16] C. Reinhardt, T. Müller, A. Bourassa, and J. C. Sankey, "Ultralow-noise SiN trampoline resonators for sensing and optomechanics," Phys. Rev. X, vol. 6, no. 2, p. 021001, 2016, doi: 10.1103/PhysRevX.6.021001.

[17] M. J. Weaver et al., "Nested trampoline resonators for optomechanics," Appl. Phys. Lett., vol. 108, no. 3, 033501, 2016, doi: $10.1063 / 1.4939828$

[18] L. G. Villanueva and S. Schmid, "Evidence of surface loss as ubiquitous limiting damping mechanism in SiN micro- and nanomechanical resonators," Phys. Rev. Lett., vol. 113, no. 22, p. 227201, 2014, doi: 10.1103/PhysRevLett.113.227201.

[19] A. Borrielli et al., "Control of recoil losses in nanomechanical SiN membrane resonators," Phys. Rev. B, Condens. Matter, vol. 94, no. 12, 2016, Art. no. 121403 , doi: 10.1103/PhysRevB.94.12140.

[20] E. Serra et al., "Microfabrication of large-area circular high-stress silicon nitride membranes for optomechanical applications," AIP Adv, vol. 6, no. 6, 2016, Art. no. 065004, doi: 10.1063/1.4953805.

[21] P.-L. Yu, T. P. Purdy, and C. A. Regal, "Control of material damping in high- $Q$ membrane microresonators," Phys. Rev. Lett., vol. 108, no. 8 , 2012, Art. no. 083603, doi: 10.1103/PhysRevLett.108.083603.

[22] M. P. Morse, Vibration and Sound. New York, NY, USA: McGraw-Hill, 1948, pp. 133-147.

[23] P. R. Saulson, "Thermal noise in mechanical experiments," Phys. Rev. D, Part. Fields, vol. 42, no. 8, p. 2437, 1990, doi: 10.1103/PhysRevD.42.2437.

[24] L. Meirovitch, Dynamics and Control of Structures Hoboken, NJ, USA: Wiley, 1990

[25] Y. Levin, "Internal thermal noise in the LIGO test masses: A direct approach," Phys. Rev. D, Part. Fields, vol. 57, no. 2, p. 659, 1998, doi: 10.1103/PhysRevD.57.659.

[26] X. Liu, T. H. Metcalf, Q. Wang, and D. M. Photiadis, "Elastic properties of several silicon nitride films," in Proc. Mater. Res. Soc. Symp., vol. 989, 2007, p. A22-01, doi: 10.1557/PROC-0989-A22-01.

[27] E. Serra et al., "A 'low-deformation mirror' microoscillator with ultra-low optical and mechanical losses," Appl. Phys. Lett., vol. 101, no. 7, 2012, Art. no. 071101, doi: $10.1063 / 1.4745510$.

[28] A. Borrielli et al., "Low-loss optomechanical oscillator for quantumoptics experiments," Phys. Rev. Appl., vol. 3, no. 5, 2015, Art. no. 054009, doi: 10.1103/PhysRevApplied.3.054009.

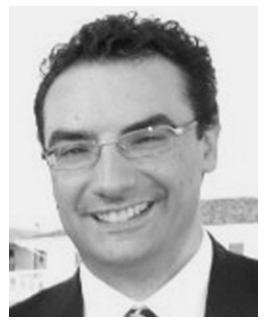

Enrico Serra received the degree in electrical engineering from the University of Padua, in 1999, and the Ph.D. degree in industrial and information engineering from the University of Udine, Italy, in 2003. During the Ph.D. degree, he was involved in the evaluation of the electromagnetic behaviors of multistrand cable-in-conduit cables and joints used for fusion energy (ITER Tokamak nuclear fusion reactor) and particle physics research. He joined the Centre for Material and Microsystems of the Fondazione Bruno Kessler in collaborating for the development of transducers for high-precision measurements (ground-based gravitational wave detectors) in 2004 and in the Cleanroom Facility as a Designer and a Process Engineer of MEMS sensors and silicon solar cells for concentration photovoltaics. Since 2013, he has been a Research Associate with the Delft Institute of Microsystems and Nanoelectronics, Delft University of Technology. He has been working at the INFN-TIFPA since 2015 coordinating the project HUMOR that aims at exploring possible effects of quantum gravity with opto-mechanical oscillators. He is currently involved in developing low-loss MEMS and micro-optomechanical-microsystems devices for fundamental physics experiments in quantum optics, and non-equilibrium properties of thermal noise.

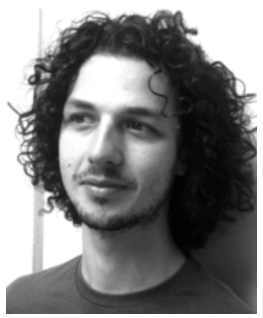

Bruno Morana received the M.Sc. degree in electronic engineering from the Polytechnic of Milan, Milan, Italy, in 2007 . He is currently pursuing the $\mathrm{Ph} . \mathrm{D}$. degree with the Laboratory of Electronic Components Technology and Materials, with a focus on the fabrication of surface micromachined nanoreactors made of silicon carbide. During the master's thesis, he joined the Electronic Technology Department, Polytechnic University of Madrid, Madrid, Spain, where he was a Researcher. His master's thesis and research activity focused on the fabrication and characterization of $\mathrm{Si}$ and $\mathrm{SiGe}$ nanocrystals embedded in $\mathrm{SiO} 2$ thin films. In 2008, he joined the Laboratory of Electronic Components Technology and Materials, Delft University of Technology, Delft, The Netherlands, as a Researcher. He focused on the development and optimization of procedures for the ultra-high vacuum compatible assembly of MEMS nanoreactors. His research interests include the fabrication and characterization of thin films, and the development of fabrication technologies for MEMS devices.

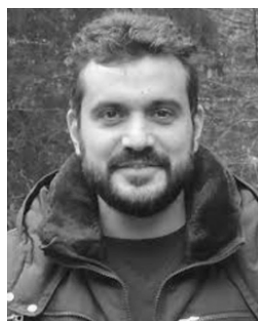

Antonio Borrielli was born in Reggio Calabria, Italy, in 1979. He received the M.S. and the Ph.D. degrees in physics from the University of Messina, Italy, in 2004 and 2009, respectively. He is currently a Researcher with the Institute of Materials for Electronics and Magnetism, Consiglio Nazionale delle Ricerche, Trento, Italy. His main research activities are focused on the design and realization of low-loss mechanical oscillators and their uses in fundamental physics experiments in the fields of thermal noise properties and dissipation phenomena related to non-equilibrium systems, quantum optomechanics, and tests of quantum gravity theories. 


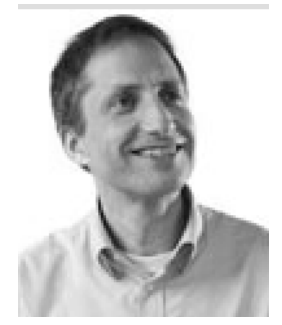

Francesco Marin received the Ph.D. degree in physics from the Scuola Normale Superiore of Pisa in 1994. He was Post-Doctoral Researcher with Laboratoire Kastler-Brossel, Paris, and INO, Florence, and a permanent position as a Researcher with the University of Florence in 1998. He has been an Associate Professor with the Dipartimento di Fisica e Astronomia and LENS, Università di Firenze, since 2002. He has co-authored over 100 papers in peer-reviewed scientific journals. He coordinated the European RTN VCSELs for the Information Society Technology Applications Project, under Contract HPRN-CT-2000-00034, and the MIUR QUANTOM Project. He has been a Local Scientist in-charge of the INFN Projects, including AURIGA, DUAL, and SQUALO, and for a MIUR Prin Project from 2010 to 2011. He is currently a Spokesman of the INFN Project under Grant HUMOR, aiming at exploring possible effects of quantum gravity with opto-mechanical oscillators, and coordinates the European QuantERA Project QuaSeRT (optomechanical quantum sensors at room temperature). His research interests include atomic physics, quantum optics, laser physics, nonlinear and stochastic dynamics, and gravitational waves detection.

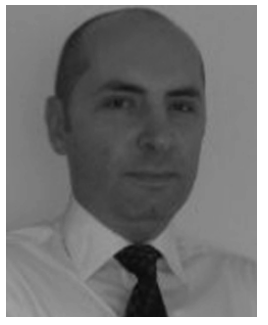

Gregory Pandraud received the Ph.D. degree in optics, optoelectronics from the University of SaintEtienne, France, in 1998. He then joined the University of Twente, The Netherlands, as a Post-Doctoral Fellow for one year. His work focused on optical sensors for TAS devices. From 1999 to 2002, he was a Development Engineer with Bookham Technologies plc., U.K., and the Senior Design Manager with Opsitech S.A., France, where he developed integrated optical components for DWDM and next generation networks applications. He joined the Delft University of Technology in 2003, after working as a Senior Researcher. $\mathrm{He}$ is currently the Project Manager with the DIMES Technology Center

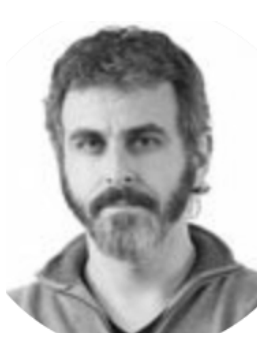

Antonio Pontin received the Laurea and Ph.D. degrees from the University of Trento, Italy, in 2010 and 2014, respectively. From 2014 to 2016, he was a Post-Doctoral Fellow with the Dipartimento di Fisica e Astronomia and LENS, Università di Firenze. In 2016, he moved to the University College London, London, as a Research Associate. He has participated in the HUMOR Project, investigating possible deviations of the Heisenberg uncertainty principle as predicted by models in the quantum gravity framework. His main research activities focus on the study of the optomechanical interaction exploiting both clamped and levitated oscillators. He is currently a part of testing the large-scale limit of quantum mechanics collaboration that aims to implement tests of the quantum superposition principle on macroscopic objects. In 2017, he received the Marie Sklodowka-Curie Individual Fellowship.

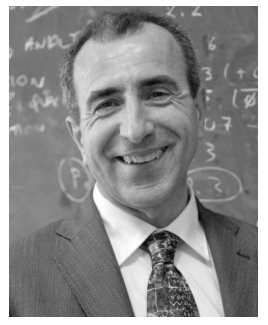

Giovanni Andrea Prodi was contributing to the development of gravitational wave detectors and of related data analysis methodologies for the last25 years. He is currently an Associate Professor in experimental physics with the Università di Trento. He is also the Coordinator of the data analysis activities of the Virgo Collaboration and the Co-Chair of the Joint Data Analysis Council with the LIGO Scientific Collaboration. His research group gave a direct contribution to the first detection of gravitational waves. He is currently participating in experimental validations of the Heisenberg uncertainty principle against the possible deviations predicted by the quantum models of gravity. His scientific interests include experiments on quantum noise limits to precision measurements.

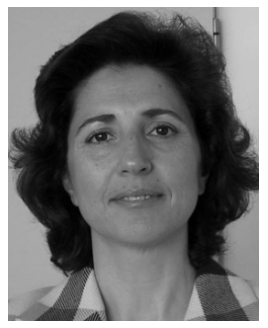

Pasqualina M. Sarro (F'06) received the Laurea degree (cum laude) in solid-states physics from the University of Naples, Italy, in 1980, and the $\mathrm{Ph} . \mathrm{D}$. degree in electrical engineering from the Delft University of Technology, The Netherlands, in 1987. From 1981 to 1983 , she was a Post-Doctoral Fellow with the Division of Engineering, Photovoltaic Research Group, Brown University, Rhode Island, USA. Since then, she has been with the Delft Institute of Microsystems and Nanoelectronics, Delft University of Technology, where she has been responsible for research on integrated silicon sensors and MEMS technology. In 2001, she became an A. van Leeuwenhoek Professor. Since 2004, she has been the Head of the Electronic Components, Materials, and Technology Laboratory. Since 2009, she has been the Chair of the Microelectronics Department, Delft University. She has authored or co-authored over 400 journals and conference papers. In 2006, she became a member of the Royal Netherlands Academy of Arts and Sciences . She is a member of the Technical Program Committee and the International Steering Committee for several international conferences, including the IEEE MEMS, the IEEE Sensors, Eurosensors, and Transducers. In 2006, she was elected as an IEEE Fellow for her contribution to micromachined sensors, actuators, and microsystems. In 2004, she received the EUROSENSORS Fellow Award for her contribution to the field of sensor technology. He is also the Technical Program Co-Chair of the First IEEE Sensors 2002 Conference and the Technical Program Chair of the Second and Third IEEE Sensors Conference in 2003 and 2004 and the General Co-Chair of the IEEE MEMS 2009.

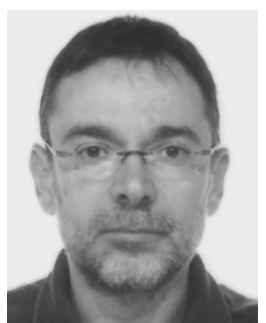

Michele Bonaldi received the Ph.D. degree in physics from the University of Padua in 1990, with a thesis on superfluid helium. He is currently a Senior Researcher with the Institute of Materials for Electronics and Magnetism, Trento (laboratory established through a joint effort of the Consiglio Nazionale delle Ricerche and the Fondazione Bruno Kessler). His research interests range from precision measurements with micro-mechanical and opto-mechanical devices to fundamental physics experiments (gravitational wave detectors, non-equilibrium properties of thermal noise, and mechanical quantum resonators) 